\title{
Estimation of pilot proficiency during simulator trainings: An auditory steady-state response study
}

\author{
Aki TSURUHARA (tsuruahra3885@inet.asdf.mod.go.jp) ${ }^{1}$, Shunsuke HAYASHI ${ }^{1}$, Masashi ARAKE ${ }^{1}$, \\ Yusuke YOKOTA ${ }^{2}$, Yasushi NARUSE ${ }^{2}$, Akio HASHIMOTO ${ }^{1}$ \\ ${ }^{1}$ Aeromedical laboratory, Japan Air Self-Defense Force, Ministry of Defense, Tachikawa 1-2-10, Tokyo, Japan \\ ${ }^{2}$ Center for Information and Neural Networks, National Institute of Information and Communications Technology, \\ Iwaoka 588-2, Nishi-ku, Kobe-city, Hyogo, Japan
}

\begin{abstract}
Estimation of pilot proficiency is important to provide efficient and effective training and to prevent flight accidents. We hypothesized that pilot proficiency could be estimated by assessing mental workload during flight training, and auditory steady-state response (ASSR), a kind of auditory evoked brain potential, could be an index of mental workload during flight training in a simulator, consequently, an index of pilot proficiency. A periodic sound induces ASSRs that have the peak frequency at the frequency of the sound, and reducing attention to the sound is known to decrease amplitudes of ASSRs. Then, when a task-irrelevant sound induced ASSRs during a task phase, such as take-off or landing, in flight training, smaller ASSRs would indicate limited attention to the task-irrelevant sound and greater attention to the task. That is, smaller ASSRs could imply the workload was high. Thus, ASSRs during a task phase would be small when a pilot is a novice and become larger with the pilot getting trained. In this case, ASSRs could be used to estimate pilot proficiency. In this study, three participants with no flight experience underwent a series of simulator training sessions, each of that lasted approximately two hours. During taxing, take-off, level flight and landing task phases in six training sessions, a task-irrelevant sound of the frequency of $40 \mathrm{~Hz}$ was presented and electroencephalogram (EEG) of each participant was recorded. The results showed that the ASSRs were getting larger with the participants experienced more training sessions. The results of this study indicate that ASSRs could be used to estimate pilot proficiency.
\end{abstract}

Keywords: Auditory steady-state response, pilot proficiency, mental workload, flight training

\section{Introduction}

Estimation of pilot proficiency is important to provide efficient and effective training and to prevent flight accidents. Mental workload during a task phase, such as take-off or landing, would be higher when a pilot is a novice and become lower with the pilot getting trained. Accordingly, pilot proficiency could be estimated by assessing mental workload during flight training. This study examined whether auditory steady-state response (ASSR), a kind of auditory evoked brain potential, could be used to assess mental workload during flight training in a simulator, and to estimate pilot proficiency. A periodic sound induces ASSRs that have the peak frequency at the frequency of the sound, and it has been shown that reducing attention to the sound decreases peak amplitude of the ASSRs (Roth et al., 2013). Then, when a task-irrelevant sound induced ASSRs during a task phase in flight training, smaller ASSRs would indicate limited attention was provided for the task-irrelevant sound and greater attention was provided for the task. That is, smaller ASSRs could imply the workload was high. Thus, we hypothesized that ASSRs during a task phase, such as landing, would be small when a pilot is a novice and become larger with the pilot getting trained. In this case, ASSRs could be used to estimate pilot proficiency.

\section{Method}

\subsection{Participants}

Three technical officials of Japan Air Self-Defense Force (JASDF) participated a series of flight training in a 
simulator. All participants had no experience of flight trainings in simulators or in real aircraft before this study. An experienced pilot prepared the training syllabus and monitored all trainings as an instructor.

\subsection{Procedure}

The participants underwent eight training sessions, each of that lasted approximately two hours. During taxing, take-off, level flight and landing task phases in the six training sessions, a task-irrelevant sound of the frequency of $40-\mathrm{Hz}$ was presented binaurally to the participants with the headset in the simulator, and electroencephalogram (EEG) at $\mathrm{FC} 3, \mathrm{FC} 4, \mathrm{FCz}$ and $\mathrm{Oz}$ (Figure 1) of each participant was measured in the sampling rate of 1000 Hz. In the other two sessions the participants experienced additional tasks and EEG was not measured. The results of EEG were not told to the participants until they completed all eight training sessions. After finishing every training session, the instructor gave feedback on the flight skills to each participant.

Figure 1. Sensor positions to measure EEG

\subsection{Analysis}

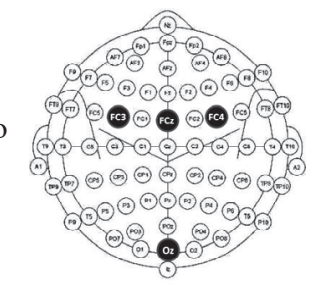

The EEG data of each task phase of each participant was band-pass filtered between 1.0 and $100 \mathrm{~Hz}$ and divided into segments of $8192 \mathrm{~ms}$ (8192 sampled data) without overlapping, and a Fast Fourier Transform (FFT) algorithm was applied to each segment. The signal-to-noise ratio (SNR) of the amplitude at each electrode was then computed. The SNR is the ratio of the amplitude at the frequency of interest to the averaged amplitude of 20 neighboring frequencies, and clearly distinguish the response of interest from noise (Rossion et al., 2012). The SNRs of all segments were averaged, and we used the SNRs at the closest frequency to the stimulus frequency $(40 \mathrm{~Hz})$ as the amplitude of ASSRs.

\section{Results and Discussion}

The results showed that, for at least one of the participants, the amplitudes of the ASSRs were clearly getting larger with the participant experienced more training sessions (Figure 2). The other two participants showed the similar tendency in some task-phases. These results showed the possibility that ASSRs could be used to estimate pilot proficiency.

Compared to the other traditional methods to assess mental workload, measuring ASSRs have some advantages in applying to the flight situation. Questionnaires, physiological measures, such as event-related potentials (ERPs) and heart rate variability, and the secondary-task method have been used to assess mental workload. These measurements, however, have some limits to use in the flight situation. Questionnaires depend on subjective and retrospective description, ERPs are easily affected by noise, heart rate variability is not sensitive enough to mental workload in many cases, and the secondary-task method could interfere with the primary task. By contrast, ASSRs provide an objective and non-retrospective index, are robust to the noise that induced by body movements in flight operation, have been shown to be sensitive to mental workload, and measuring EEG itself does not disturb the primary task. Though we had to consider that the voice instructions from air-traffic controllers could interfere with the sound necessary to elicit ASSRs, ASSRs would be useful to estimate pilot proficiency at least in some task phases.

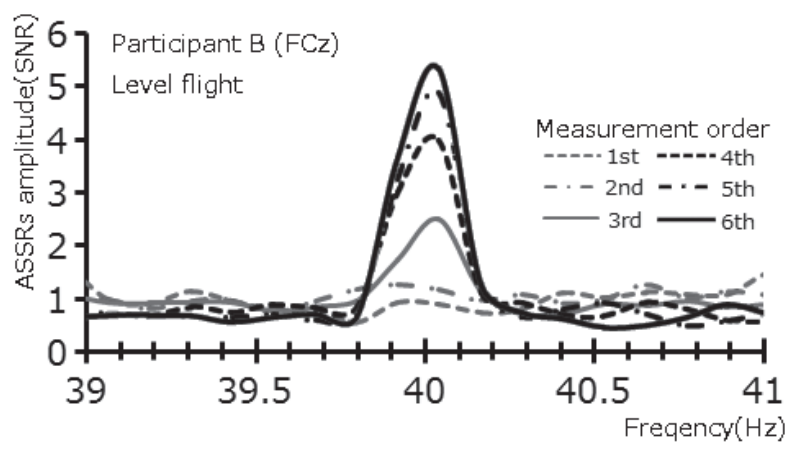

Figure 2. ASSRs change along with expertise (typical example)

\section{References}

Roth, C. Gupta, C.N., Plis, S.M., Damaraju, E., Khullar, S. Calhoun, V.D. and Bridwell, D.A., The influence of visuospatial attention on unattended auditory $40 \mathrm{~Hz}$ responses, Frontiers in Human Neuroscience, 7:370, 2013.

Rossion, B., Prieto, E.A., Boremanse, A., Kuefner, D. and Van Belle, G., A steady-stage visual evoked potential approach to individual face perception: effect of inversion, contrast-reversal and temporal dynamics, Neuroimage, 63, 1585 - 1560, 2012. 